\title{
ZERO-FREE PARABOLIC REGIONS FOR POLYNOMIALS WITH COMPLEX COEFFICIENTS
}

\author{
HANS-J. RUNCKEL
}

\begin{abstract}
Recent results by P. Henrici, E. B. Saff and R. S. Varga on zero-free parabolic regions for sequences of polynomials generated from three-term recurrence relations with real coefficients are generalized to complex coefficients by continued fraction methods. Especially, it is shown that all zeros of the generalized Bessel polynomials $Y_{n}^{(\delta)}$ for complex $\delta$ are contained in a cardioid region, which generalizes a result of E. B. Saff and R. S. Varga for real $\delta$.
\end{abstract}

1. Introduction. In [6] E. B. Saff and R. S. Varga showed that certain sequences of polynomials defined by three-term recurrence relations with real coefficients have no zeros in a parabolic region. From this it is deduced (cf. [2, pp. 75-89]) that all zeros of the $m$ th generalized Bessel polynomial $Y_{m}^{(\delta)}(z)$, depending on the parameter $\delta$, are contained in the cardioid region $\left\{z=r e^{i \vartheta} \in \mathbf{C}: 0<r<(1+\cos \vartheta) /(m+1+\delta),|\vartheta|<\pi\right\} \cup\{2 /(m+1+\delta)\}$, provided $m+1+\delta>0$. In [3] P. Henrici generalized the results of [6] so as to apply to interpolation polynomials with real interpolation points. In the present paper the continued fraction method of P. Henrici [3] is generalized so as to yield zero-free parabolic regions also for polynomials satisfying three-term recursion relations with complex coefficients, which include interpolation polynomials with complex interpolation points. As an interesting application, it is shown that all zeros of $Y_{m}^{(\delta)}(z), m \geqslant 2$, are contained in the open cardioid region

$$
\left\{z=r e^{i \vartheta} \in \mathbf{C}: 0<r<(1+\cos \vartheta) /|m+1+\delta| \cos ^{2}(\chi / 2),|\vartheta|<\pi\right\},
$$

where $m+1+\delta=|m+1+\delta| e^{i \chi} \neq 0$ and $|\chi|<\pi$, i.e. $\delta$ is complex, provided $m+1+\delta$ is not $\leqslant 0$. In particular, for $0<|\chi|<\pi / 2$ this region is properly contained in the open cardioid region

$$
\left\{z=r e^{i \vartheta} \in \mathbf{C}: 0<r<(1+\cos \vartheta) /(m+1+\operatorname{Re} \delta),|\vartheta|<\pi\right\} .
$$

2. The basic continued fraction method. More generally than in [3] we define

$$
\begin{aligned}
q_{n}:=\left(b_{n}+z_{n+1}\right) q_{n-1}-a_{n} z_{n} q_{n-2}, \quad n \in \mathbf{N}, q_{-1}:=0, q_{0}:=1, \\
\quad \text { where } a_{n}, b_{n}, z_{n} \in \mathbf{C}, n \in \mathbf{N} \text { and } a_{n}=\left|a_{n}\right| e^{i \psi_{n}} \neq 0, n \geqslant 2 .
\end{aligned}
$$

Since $q_{-1}=0$, all $q_{n}, n \geqslant 1$, are independent of $a_{1}$ and $z_{1}$. We want to determine conditions on $a_{n}, b_{n}, z_{n}$ which imply $q_{n} \neq 0$ for all $n \in \mathbf{N}$ or $1 \leqslant n \leqslant N$. Similarly as

Received by the editors March 27, 1982.

1980 Mathematics Subject Classification. Primary 30C 15; Secondary, 30B70. 
in [3] we observe that $q_{n}$ is the denominator of the continued fraction

$$
w_{n}=\frac{p_{n}}{q_{n}}=\frac{1}{b_{1}+z_{2}}-\frac{z_{2} a_{2}}{b_{2}+z_{3}}-\cdots-\frac{z_{n} a_{n}}{b_{n}+z_{n+1}} .
$$

Since $p_{n}, q_{n}$ cannot vanish simultaneously if all $a_{n} z_{n} \neq 0$, we first assume $z_{n} \neq 0$ for $n \geqslant 2$. Later on we will see that all results below remain valid if some $z_{n}=0$. It then suffices to show that $w_{n} \neq \infty$ in order to obtain $q_{n} \neq 0$. (2) can be written as $w_{n}=s_{1} \circ \cdots \circ s_{n}\left(z_{n+1}\right)$, where $(\overline{\mathbf{C}}$ is the closed complex plane $)$

$$
\begin{aligned}
& s_{n}(u):=z_{n}\left(1-\left(a_{n} /\left(b_{n}+u\right)\right)\right), \quad n \geqslant 2, \quad \text { and } \\
& s_{1}(u):=1 /\left(b_{1}+u\right), \quad u \in \overline{\mathbf{C}} .
\end{aligned}
$$

We want to determine closed half-planes $H_{n} \subset \overline{\mathbf{C}}, n \in \mathbf{N}$, and (large) sets $P_{n} \subset \mathbf{C}$, $n \geqslant 2$, such that for $z_{n} \in P_{n}$,

$$
D_{n}:=s_{n}\left(H_{n}\right) \subset H_{n-1} \text { holds and } D_{n} \subset \mathrm{C} \text { is a finite closed disk, } n \geqslant 2 \text {. }
$$

Assume, in addition, that $-b_{1} \notin H_{1}$ (or more generally $-b_{1} \notin D_{2}$ ). Then (cf. (8)) $z_{n} \in P_{n}, n \geqslant 2$, and (4) imply $w_{n} \neq \infty$ and, hence, $q_{n} \neq 0$ for $n \geqslant 1$. We now define, for $n \in \mathbf{N}$,

$$
H_{n}:=\left\{\zeta \in \mathbf{C}: \operatorname{Re} e^{i \varphi_{n}}\left(\zeta+d_{n}\right) \geqslant 0\right\}, \quad \varphi_{n} \in \mathbf{R}, d_{n} \in \mathbf{C} ; \operatorname{Re} e^{i \varphi_{n}} d_{n}>0 .
$$

Since $s_{n}\left(-b_{n}\right)=\infty, D_{n}$ is a finite disk iff $-b_{n} \notin H_{n}$ or $\operatorname{Re} e^{i \varphi_{n}}\left(b_{n}-d_{n}\right)>0$, which we want to assume for $n \geqslant 2$. Next, we evaluate $D_{n}$ explicitly. Let $b_{n}^{*}$ be symmetric to $-b_{n}$ with respect to $\partial H_{n}$, the boundary of $H_{n}$. Then $b_{n}^{*}=-d_{n}+e^{-2 i \varphi_{n}}\left(\bar{b}_{n}-\bar{d}_{n}\right)$, and the center of $D_{n}$ is given by $\xi_{n}:=s_{n}\left(b_{n}^{*}\right)=z_{n}\left(1-c_{n}\right)$, where

$$
c_{n}:=a_{n} /\left(b_{n}+b_{n}^{*}\right)=e^{i \varphi_{n}} a_{n} / 2 \operatorname{Re} e^{i \varphi_{n}}\left(b_{n}-d_{n}\right), \quad n \geqslant 2 .
$$

Since $z_{n}=s_{n}(\infty) \in \partial D_{n}$, the boundary of $D_{n}$, the radius of $D_{n}$ is given by $r_{n}:=\left|z_{n}-\xi_{n}\right|=\left|z_{n} c_{n}\right|$. Hence

$$
D_{n}=\left\{\zeta \in \mathbf{C}:\left|\zeta-z_{n}+z_{n} c_{n}\right| \leqslant\left|z_{n} c_{n}\right|\right\}, \quad n \geqslant 2 .
$$

For $n \geqslant 2, D_{n} \subset H_{n-1}$ is equivalent to $\operatorname{Re} e^{i \varphi_{n-1}}\left(\xi_{n}+d_{n-1}\right)-r_{n} \geqslant 0$ or, explicitly,

$$
\operatorname{Re} e^{i \varphi_{n-1}}\left(z_{n}-z_{n} c_{n}+d_{n-1}\right)-\left|z_{n} c_{n}\right| \geqslant 0
$$

or

$$
\operatorname{Re} e^{i \varphi_{n-1}}\left(z_{n}+d_{n-1}\right) \geqslant \operatorname{Re}\left(e^{i \varphi_{n-1}} z_{n} c_{n}\right)+\left|z_{n} c_{n}\right|, \quad n \geqslant 2 .
$$

Since here the right side is $\geqslant 0, z_{n} \in H_{n-1}$ follows and, hence, $P_{n} \subset H_{n-1}$ must hold for $n \geqslant 2$. We now define $P_{n}$ by

$$
\begin{aligned}
& P_{n}:=\left\{\zeta \in \mathbf{C}: \operatorname{Re}\left(e^{i \varphi_{n-1}} \zeta c_{n}\right)+\left|\zeta c_{n}\right| \leqslant 2 h\left(c_{n}\right) \operatorname{Re} e^{i \varphi_{n-1}}\left(\zeta+d_{n-1}\right)\right\}, \\
& \text { where the real-valued function } h \text { satisfies } 0<h\left(c_{n}\right) \leqslant 1 / 2, n \geqslant 2 .
\end{aligned}
$$

Obviously (8) holds for each $z_{n} \in P_{n}$, and different choices of the function $h$ lead to different conditions on $a_{n}, b_{n}, z_{n}$. Next, $a_{n} \neq 0$ implies $c_{n} \neq 0$. Hence (9) is 
equivalent to

$$
\begin{aligned}
P_{n}=\left\{\zeta \in \mathrm{C}:|\zeta| \leqslant \operatorname{Re}\left(e ^ { i \varphi _ { n - 1 } } \zeta \left(2 h\left(c_{n}\right)\right.\right.\right. & \left.\left.-c_{n}\right) /\left|c_{n}\right|\right) \\
& \left.+2\left(h\left(c_{n}\right) /\left|c_{n}\right|\right) \operatorname{Re} e^{i \varphi_{n-1} d_{n-1}}\right\},
\end{aligned}
$$

where $P_{n} \subset H_{n-1}, n \geqslant 2$. Observe that each $P_{n}$ is convex. Finally, assume that $z_{1}, \ldots, z_{m} \neq 0, \quad z_{m+1}=\cdots=z_{m+j}=0, \quad z_{m+j+1} \neq 0$ for some $m, j \geqslant 1$. Then $q_{1}, \ldots, q_{m} \neq 0$ by the preceding considerations and, by (1),

$$
q_{m+\nu}=\left(b_{m+\nu}+z_{m+\nu+1}\right) q_{m+\nu-1}, \quad 1 \leqslant \nu \leqslant j,
$$

which is $\neq 0$ because $z_{m+\nu+1} \in P_{m+\nu+1} \subset H_{m+\nu}$ and $-b_{m+\nu} \notin H_{m+\nu}$. For $n \in \mathbf{N}$ then $q_{m+j+n-1}=q_{m+j-1} q_{n}^{*}$ holds, where $q_{n}^{*}$ satisfies

$$
q_{n}^{*}=\left(b_{(m+j-1)+n}+z_{(m+j-1)+n+1}\right) q_{n-1}^{*}-a_{(m+j-1)+n} z_{(m+j-1)+n} q_{n-2}^{*}
$$

with $q_{-1}^{*}=0, q_{0}^{*}=1$. To these $q_{n}^{*}$ the above considerations can be applied until, again for some $n, z_{(m+j-1)+n}=0$ holds. But then the preceding argument can be repeated.

3. General results. In the preceding considerations we have proved

THEOREM 1. If (cf. (1), (5), (9)) $\operatorname{Re} e^{i \varphi_{n}}\left(b_{n}-d_{n}\right)>0,1 \leqslant n \leqslant N$ and $z_{n} \in P_{n}$, $2 \leqslant n \leqslant N+1$, or if, more generally, $\left|b_{1}+z_{2}-z_{2} c_{2}\right|>\left|z_{2} c_{2}\right|$ (i.e. $-b_{1} \notin D_{2}$ ), $\operatorname{Re} e^{i \varphi_{n}}\left(b_{n}-d_{n}\right)>0,2 \leqslant n \leqslant N$ and $z_{n} \in P_{n}, 3 \leqslant n \leqslant N+1$, then $q_{n} \neq 0$ for $1 \leqslant n$ $\leqslant N$. If the above conditions hold for arbitrary $N \geqslant 2$, then $q_{n} \neq 0$ for $n \geqslant 1$.

Next, we assume that $z_{n+1} \in \partial H_{n} \cap \partial P_{n+1}$ for some $n \in \mathbf{N}$. Then (9) implies $\operatorname{Re} e^{i \varphi_{n}} z_{n+1} c_{n+1}+\left|c_{n+1} z_{n+1}\right|=0$. Hence, $\operatorname{Im} e^{i \varphi_{n}} z_{n+1} c_{n+1}=0$ and, from (6), we obtain

$$
z_{n+1}=-e^{-i\left(\varphi_{n}+\varphi_{n+1}+\psi_{n+1}\right)}\left|z_{n+1}\right| .
$$

This and $z_{n+1} \in \partial H_{n}$ imply

$$
\operatorname{Re} e^{i \varphi_{n}} d_{n}=-\operatorname{Re} e^{i \varphi_{n}} z_{n+1}=\left|z_{n+1}\right| \cos \left(\varphi_{n+1}+\psi_{n+1}\right) .
$$

Hence, $\cos \left(\varphi_{n+1}+\psi_{n+1}\right)>0$ since $\operatorname{Re} e^{i \varphi_{n}} d_{n}>0$. If $\left|z_{n+1}\right|$ from (12) is substituted into (11), we obtain

$$
z_{n+1}=z_{n+1}^{\prime}:=\frac{-e^{-i\left(\varphi_{n}+\varphi_{n+1}+\psi_{n+1}\right)} \operatorname{Re} e^{i \varphi_{n}} d_{n}}{\cos \left(\varphi_{n+1}+\psi_{n+1}\right)}, \quad \cos \left(\varphi_{n+1}+\psi_{n+1}\right)>0 .
$$

$\left(z_{n+1}^{\prime}=-d_{n}\right.$ if $\varphi_{n}=\varphi_{n+1}=0, a_{n+1}>0, d_{n}>0$.)

Conversely, one verifies that $z_{n+1}^{\prime} \in \partial H_{n} \cap \partial P_{n+1}$ holds. Therefore, $\partial H_{n}$ and $\partial P_{n+1}$ always have exactly one point, $z_{n+1}^{\prime}$, in common, provided

$$
\cos \left(\varphi_{n+1}+\psi_{n+1}\right)>0 \text {. }
$$

If, in particular, $-b_{\nu} \notin H_{\nu}, z_{\nu} \in P_{\nu}, \nu \geqslant 2$, and $-b_{1} \in \partial H_{1}$, then $w_{n}=\infty$ or $s_{2} \circ \cdots \circ s_{n}\left(z_{n+1}\right)=-b_{1}$ can occur for some $n \geqslant 2$ only if $-b_{1} \in \partial D_{2}$ also. By the above considerations this is only possible if $z_{n+1}=z_{n+1}^{\prime}$ in (13) and if all disks

$$
D_{2}=s_{2}\left(H_{2}\right) \supset s_{2} \circ s_{3}\left(H_{3}\right) \supset \cdots \supset s_{2} \circ \cdots \circ s_{n}\left(H_{n}\right)
$$

touch $\partial H_{1}$ at the point $-b_{1}$. 
Finally, we assume that $z_{n}=z, H_{n}=H:=\left\{\zeta \in \mathbf{C}: \operatorname{Re} e^{i \varphi}(\zeta+d) \geqslant 0\right\}, n \geqslant 1$, with fixed $\varphi \in \mathbf{R}, d \in \mathbf{C}$ and $-b_{1} \in \partial H,-b_{\nu} \notin H, \nu \geqslant 2$. If for some $n \geqslant 2$ and $z \in \cap_{\nu=2}^{n+1} P_{\nu}, s_{2} \circ \cdots \circ s_{n}(z)=-b_{1}$ holds, then $-b_{1} \in \partial D_{2}$ also and, hence, $z=z_{n+1}^{\prime} \in \partial H$. Now $-b_{1} \in \partial H \cap \partial D_{2}$ is equivalent to $\operatorname{Re} e^{i \varphi}\left(b_{1}-d\right)=0$ and $\left|b_{1}+z-z c_{2}\right|=\left|z c_{2}\right|$. If $\operatorname{Im} e^{i \varphi}\left(b_{1}+z-z c_{2}\right) \neq 0$, then

$$
\left|b_{1}+z-z c_{2}\right|>\operatorname{Re} e^{i \varphi}\left(b_{1}+z-z c_{2}\right)=-\operatorname{Re} e^{i \varphi} z c_{2}=\left|z c_{2}\right|
$$

because $\operatorname{Re} e^{i \varphi}\left(b_{1}+z\right)=\operatorname{Re} e^{i \varphi}(d+z)=0$ and (9), since $z \in \partial H \cap \partial P_{2}$. But this contradicts $-b_{1} \in \partial D_{2}$. Hence, $\operatorname{Im} e^{i \varphi}\left(b_{1}+z-z c_{2}\right)=0$. This yields $\operatorname{Im} e^{i \varphi}\left(b_{1}+z\right)$ $=0$, since $-\operatorname{Re} e^{i \varphi} z c_{2}=\left|z c_{2}\right|$ implies $\operatorname{Im} e^{i \varphi} z c_{2}=0$. Therefore $b_{1}+z=0$. If $n=2$, then this, $0=b_{1}+s_{2}(z)$, and $z \neq 0$ imply $b_{2}+z=\infty$. If $n \geqslant 3$, then $b_{1}+z=0$, $z \neq 0$, and $0=b_{1}+s_{2} \circ \cdots \circ s_{n}(z)$ imply $s_{3} \circ \cdots \circ s_{n}(z)=\infty$. But this is impossible by applying Theorem 1 to the denominators of the continued fraction

$$
\frac{1}{b_{3}+z}-\frac{z a_{4}}{b_{4}+z}-\cdots
$$

since $-b_{n} \notin H$ for $n \geqslant 2$. Hence, $q_{n} \neq 0$ for $n \geqslant 2$ and $q_{1}=0$ iff $z=-b_{1}$. We thus have proved

THEOREM 2. (1) If (cf. (1),(5), (9), (13)) $\operatorname{Re} e^{i \varphi_{1}}\left(b_{1}-d_{1}\right)=0$ (i.e. $-b_{1} \in \partial H_{1}$ ), $\operatorname{Re} e^{i \varphi_{n}}\left(b_{n}-d_{n}\right)>0,2 \leqslant n \leqslant N$ and if $z_{n} \in P_{n}, 2 \leqslant n \leqslant N+1$, then $q_{N} \neq 0$ provided $z_{N+1} \neq z_{N+1}^{\prime}$ in case $\left|b_{1}+z_{2}-z_{2} c_{2}\right|=\left|z_{2} c_{2}\right|$ (i.e. $\left.-b_{1} \in \partial D_{2}\right)$.

(2) If $\operatorname{Re} e^{i \varphi_{1}}\left(b_{1}-d_{1}\right)=0, \operatorname{Re} e^{i \varphi_{n}}\left(b_{n}-d_{n}\right)>0, n \geqslant 2$ and $z_{n} \in P_{n}, n \geqslant 2$, then $q_{n} \neq 0$ for $n \geqslant 2$ provided $z_{n+1} \neq z_{n+1}^{\prime}, n \geqslant 2$, in case $\left|b_{1}+z_{2}-z_{2} c_{2}\right|=\left|z_{2} c_{2}\right|$.

(3) Assume that all $z_{n}=z$ and that for fixed $\varphi \in \mathbf{R}, d \in \mathbf{C}, \operatorname{Re} e^{i \varphi} d>0$ holds. If $\operatorname{Re} e^{i \varphi}\left(b_{1}-d\right) \geqslant 0$, and $\operatorname{Re} e^{i \varphi}\left(b_{n}-d\right)>0,2 \leqslant n \leqslant N$, then $q_{n}(z) \neq 0$ for $z \in$ $\cap_{\nu=2}^{N+1} P_{\nu}$ and $2 \leqslant n \leqslant N .\left(q_{1}(z)=0\right.$ iff $z=-b_{1}$.) If the above conditions hold for arbitrary $N \geqslant 2$, then $q_{n}(z) \neq 0$ for $z \in \cap_{\nu=2}^{\infty} P_{\nu}$ and $n \geqslant 2$.

We now choose $h\left(c_{n}\right)=\operatorname{Re} c_{n}$ in (10). Then (10) is equivalent to

$$
P_{n}=\left\{\zeta \in \mathbf{C}:|\zeta| \leqslant \operatorname{Re}\left(e^{i \varphi_{n-1}} \zeta \bar{c}_{n} /\left|c_{n}\right|\right)+2 \operatorname{Re}\left(e^{i \varphi_{n-1}} d_{n-1}\right) \operatorname{Re} c_{n} /\left|c_{n}\right|\right\} .
$$

By (1) and (6), $\bar{c}_{n} /\left|c_{n}\right|=e^{-i\left(\varphi_{n}+\psi_{n}\right)}$. Hence,

$$
h\left(c_{n}\right)=\operatorname{Re} c_{n}>0
$$

iff $\cos \left(\varphi_{n}+\psi_{n}\right)>0$, and $\operatorname{Re} c_{n} \leqslant \frac{1}{2}$ iff $\operatorname{Re} e^{i \varphi_{n}}\left(b_{n}-a_{n}-d_{n}\right) \geqslant 0, n \geqslant 2$. If these conditions are satisfied, then $\operatorname{Re} e^{i \varphi_{n}}\left(b_{n}-d_{n}\right) \geqslant \operatorname{Re} e^{i \varphi_{n}} a_{n}>0$ holds for $n \geqslant 2$ and, hence, Theorems 1 and 2(1)(2) yield

Corollary 1. Assume that $\varphi_{n} \in \mathbf{R}, d_{n} \in \mathbf{C}$ such that $\operatorname{Re} e^{i \varphi_{n}} d_{n}>0, n \in \mathbf{N}$. Let $P_{n}, n \geqslant 2$, be the parabolic region

$$
P_{n}=\left\{\zeta \in \mathrm{C}:|\zeta| \leqslant \operatorname{Re} e^{i\left(\varphi_{n-1}-\varphi_{n}-\psi_{n}\right)} \zeta+2 \cos \left(\varphi_{n}+\psi_{n}\right) \operatorname{Re} e^{i \varphi_{n-1}} d_{n-1}\right\},
$$

where $\cos \left(\varphi_{n}+\psi_{n}\right)>0, n \geqslant 2$.

(1) If $\operatorname{Re} e^{i \varphi_{1}}\left(b_{1}-d_{1}\right)>0, \operatorname{Re} e^{i \varphi_{n}}\left(b_{n}-a_{n}-d_{n}\right) \geqslant 0,2 \leqslant n \leqslant N$, and $z_{n} \in P_{n}$, $2 \leqslant n \leqslant N+1$, or if, more generally,

$$
\left|b_{1}+z_{2}-z_{2} c_{2}\right|>\left|z_{2} c_{2}\right|
$$


$\operatorname{Re} e^{i \varphi_{n}}\left(b_{n}-a_{n}-d_{n}\right) \geqslant 0,2 \leqslant n \leqslant N$ and $z_{n} \in P_{n}, 3 \leqslant n \leqslant N+1$, then $q_{n} \neq 0$ for $1 \leqslant n \leqslant N$. If the above conditions hold for all $N \geqslant 2$, then $q_{n} \neq 0$ for $n \geqslant 1$.

(2) If $\operatorname{Re} e^{i \varphi_{1}}\left(b_{1}-d_{1}\right)=0, \operatorname{Re} e^{i \varphi_{n}}\left(b_{n}-a_{n}-d_{n}\right) \geqslant 0,2 \leqslant n \leqslant N$ and $z_{n} \in P_{n}$, $2 \leqslant n \leqslant N+1$, then $q_{N} \neq 0$ provided $z_{N+1} \neq z_{N+1}^{\prime}$ in case $\left|b_{1}+z_{2}-z_{2} c_{2}\right|=\left|z_{2} c_{2}\right|$.

(3) If $\operatorname{Re} e^{i \varphi_{1}}\left(b_{1}-d_{1}\right)=0, \operatorname{Re} e^{i \varphi_{n}}\left(b_{n}-a_{n}-d_{n}\right) \geqslant 0, n \geqslant 2$, and $z_{n} \in P_{n}, n \geqslant 2$, then $q_{n} \neq 0$ for $n \geqslant 2$ provided $z_{n+1} \neq z_{n+1}^{\prime}, n \geqslant 2$ in case $\left|b_{1}+z_{2}-z_{2} c_{2}\right|=\left|z_{2} c_{2}\right|$.

In particular, we obtain from Theorem 2(3):

COROLlaRY 2. Assume that in (1) $a_{n}=\left|a_{n}\right| e^{i \psi}$ and $b_{n}-a_{n}=\left|b_{n}-a_{n}\right| e^{i x}$ with fixed $\psi, \chi \in \mathbf{R}$ for $n \geqslant 2$. Assume also that $\rho:=\inf _{n \geqslant 2}\left|b_{n}-a_{n}\right|>0$ and put $d:=\rho e^{i \chi}$. If for some $\varphi \in \mathbf{R}, \operatorname{Re} e^{i \varphi}\left(b_{1}-d\right) \geqslant 0, \cos (\varphi+\psi)>0$ and $\cos (\varphi+\chi)$ $>0$ (i.e. $\operatorname{Re} e^{i \varphi} d>0$ ), then the polynomials $q_{n}(z)$, defined by

$$
q_{n}(z)=\left(b_{n}+z\right) q_{n-1}(z)-a_{n} z q_{n-2}(z), \quad n \in \mathbf{N}, q_{-1}:=0, q_{0}:=1,
$$

have no zeros for $n \geqslant 2$ in the closed parabolic region

$$
P^{\prime}=\left\{\zeta \in \mathbf{C}:|\zeta| \leqslant \operatorname{Re} e^{-i \psi \zeta}+2 \rho \cos (\varphi+\psi) \cos (\varphi+\chi)\right\} .
$$

$q_{1}(z)=0$ iff $z=-b_{1}$. If $\varphi=-(\chi+\psi) / 2$ and $|\chi-\psi|<\pi$, then $q_{n}(z), n \geqslant 2$, have no zeros in the closed parabolic region

$$
P=\left\{\zeta \in \mathbf{C}:|\zeta| \leqslant \operatorname{Re} e^{-i \psi} \zeta+2 \rho \cos ^{2}((\chi-\psi) / 2)\right\} \supset P^{\prime} .
$$

Proof. Since $\operatorname{Re} e^{i \varphi} a_{n}=\left|a_{n}\right| \cos (\varphi+\psi)>0$ and $\operatorname{Re} e^{i \varphi} d=\rho \cos (\varphi+\chi)>0$, Corollary 2 follows from Theorem 2(3). Observe that

$$
2 \cos (\varphi+\psi) \cos (\varphi+\chi)=\cos (2 \varphi+\psi+\chi)+\cos (\psi-\chi)
$$

is maximal for $\varphi=-(\chi+\psi) / 2$. Then

$$
\cos (\varphi+\psi)=\cos (\varphi+\chi)=\cos ((\chi-\psi) / 2)>0 \quad \text { if }|\chi-\psi|<\pi
$$

4. Application to generalized Bessel polynomials. These polynomials are defined for $n \in \mathbf{N}$ by (cf. $[2,6])$

$$
Y_{n}^{(\delta)}(z):=1+\sum_{j=1}^{n}\left(\begin{array}{l}
n \\
j
\end{array}\right)(n+\delta+1) \cdots(n+\delta+j)(-z / 2)^{j}, \quad \text { where } \delta \in \mathbf{C} .
$$

Then for fixed $m \in \mathbf{N}$ the polynomials $q_{n}^{(m+\delta)}(z):=z^{n} Y_{n}^{(m+\delta-n)}(-2 / z)$ satisfy

$$
\begin{aligned}
q_{n}^{(m+\delta)}(z)=(n+m+\delta+z) q_{n-1}^{(m+\delta)}(z)-(n-1) z q_{n-2}^{(m+\delta)}(z) & \\
& \text { with } q_{-1}^{(m+\delta)}:=0, q_{0}^{(m+\delta)}:=1 .
\end{aligned}
$$

Hence $q_{n}^{(m+\delta)}(z), n \in \dot{\mathbf{N}}$, is of type (1) with $b_{n}=n+m+\delta, a_{n}=n-1, n \in \mathbf{N}$, and, therefore, $b_{n}-a_{n}=m+1+\delta$ is independent of $n$. With the notation of Corollary 2 , we have, in this example,

$$
\psi=0, \quad \rho=|m+1+\delta| \text { and } d=m+1+\delta=|m+1+\delta| e^{i \chi}=b_{1} .
$$

If $\varphi=-\chi / 2$, then (14) yields a closed parabolic region (containing $-b_{1}$ )

$$
P=\left\{\zeta \in \mathbf{C}:|\zeta| \leqslant \operatorname{Re} \zeta+2|m+1+\delta| \cos ^{2}(\chi / 2)\right\}
$$


such that $q_{n}^{(m+\delta)}(z) \neq 0$ for $n \geqslant 2, z \in P$, provided $m+1+\delta \neq 0$ and $|\chi|<\pi$. For $0<|\chi|<\pi / 2,2 \cos ^{2}(\chi / 2)=1+\cos \chi>2 \cos \chi$ holds and, therefore, $P$ properly contains the parabolic region $P^{\prime}=\{\zeta \in \mathbf{C}:|\zeta| \leqslant \operatorname{Re} \zeta+2(m+1+\operatorname{Re} \delta)\}$.

In particular, for $n=m \geqslant 2$, this shows that $Y_{m}^{(\delta)}(z) \neq 0$ for $-2 / z \in P$. Thus we have proved

THEOREM 3. If $m+1+\delta=|m+1+\delta| e^{i \chi} \neq 0$ and $|\chi|<\pi$, then all zeros of $Y_{m}^{(\delta)}(z), m \geqslant 2$, are contained in the open cardioid region

$$
C=\left\{z=r e^{i \vartheta} \in \mathbf{C}: 0<r<(1+\cos \vartheta) /|m+1+\delta| \cos ^{2}(\chi / 2),|\vartheta|<\pi\right\} .
$$

i.e. if $m+\delta+1$ lies on the parabola $|z|+\operatorname{Re} z=2 c>0$, then all zeros of $Y_{m}^{(\delta)}(z)$, $m \geqslant 2$, are contained in

$$
C=\left\{z=r e^{i \vartheta} \in \mathbf{C}: 0<r<(1+\cos \vartheta) / c,|\vartheta|<\pi\right\} .
$$

If $0<|\chi|<\pi / 2$, then $C$ is properly contained in the open cardioid region

$$
C^{\prime}=\left\{z=r e^{i \vartheta} \in \mathbf{C}: 0<r<(1+\cos \vartheta) /(m+1+\operatorname{Re} \delta) .|\vartheta|<\pi\right\} .
$$

$Y_{1}^{(\delta)}(z)=0$ iff $z=2 /(2+\delta)$.

In the special case where $m+\delta+1$ is real and $>0$, M. G. de Bruin, E. B. Saff and R. S. Varga have proved in $[\mathbf{1}, \mathbf{5}, 7]$ that the result of Theorem 3 is sharp in the sense that each boundary point of $\left\{z=r e^{i \vartheta} \in \mathbf{C}: 0<r<(1+\cos \vartheta) / 2,|\vartheta|<\pi\right\}$ is an accumulation point of zeros of the normalized Bessel polynomials

$$
Y_{m}^{(\delta)}(2 z /(m+\delta+1)), \quad m \in \mathbf{N}, \quad \dot{m}+\delta+1>0 .
$$

Whether for nonreal $m+\delta+1$, Theorem 3 (and for nonreal $a_{n}, b_{n}$, Corollary 2 ) still is sharp is an open question. For other new results concerning the location of zeros of polynomials satisfying a three-term recurrence relation see also [4].

\section{REFERENCES}

1. M. G. de Bruin, E. B. Saff and R. S. Varga, On the zeros of generalized Bessel polynomials. I. II, Nederl. Akad. Wetensch. Indag. Math. 43 (1981), 1-25.

2. E. Grosswald, Bessel polynomials, Springer, Berlin and New York, 1978.

3. P. Henrici, Note on a theorem of Saff and Varga, Padé and Rational Approximation. Theory and Applications, Academic Press, New York, 1977, pp. 157-161.

4. E. Leopold, Approximants de Padé pour les fonctions de classes $S$, et localisation des zeros de certains polınomes, Thèse de troisième cycle, Univ. de Provence, January 18, 1982.

5. E. B. Saff and R. S. Varga, On the zeros and poles of Padé approximants to $e^{z}$, Numer. Math. 25 (1975), 1-14.

6. Zero-free parabolic regions for sequences of polynomials, SIAM J. Math. Anal. 7 (1976), $344-357$.

7. On the sharpness of theorems concerning zero-free regions for certain sequences of polynomials, Numer. Math. 26 (1976), 345-354.

Department of Mathematics, Universität Ulm, Abt Math IV, P 4066, (D79) Ulm, Federal REPUBLIC OF GERMANY 